\title{
The EnergyKids project: pilot study on the energy balance of primary school children during school days and summer camp days
}

\author{
Alice Rosi ${ }^{1}$, Cinzia Franchini ${ }^{1}$ and Francesca Scazzina ${ }^{1,2}$ \\ ${ }^{1}$ Department of Food and Drugs, University of Parma, Parma, Italy and \\ ${ }^{2}$ Giocampus Scientific Committee, Parma, Italy
}

\section{Abstract}

Specific nutritional requirements should be cover during childhood to ensure proper growth. The daily energy need is defined as the number of calories necessary to satisfy the total energy expenditure in a healthy and active organism. Since children are more inclined to be physically active during summer, their energy expenditure may vary during school days respect to summer days and a proper energy intake should be planned during both periods.

The primary aim of this study was to evaluate the energy balance during school days and summer camp days in primary school children living in the city of Parma (Italy), attending the Giocampus programme.

Participants were asked to complete a 3-day weighed food diary and to wear an activity tracker for the same 3 consecutive days twice: within a school week and a summer camp week. Height and body weight were measured at the beginning of each assessment week and BMI was calculated to define the weight status through the IOTF gender- and age-related cut-offs for children BMI. The mean energy balance was estimated as the difference between the mean total energy expenditure (calculated multiplying the daily physical activity level by the basal metabolic rate from the Schofield's predictive equation) and the mean daily energy intakes (obtained through the Italian food database of the European Institute of Oncology).

Fifty-five children $(49 \%$ F, 51\% M, 8-10 y) correctly completed the study. In both periods, the mean BMI corresponded to normal weight status, without significant differences between genders. Energy intake did not change significantly between the assessment periods or between sexes. As expected, the minutes of inactivity decreased during the summer camp period. Moreover, males were more active than females, but the increase in the total energy expenditure from school to summer camp, related to the increased physical activity, was significant in both sexes. Energy balance was negative in both assessment periods and it changed significantly from school to summer camp days, decreasing in both sexes but being more negative for boys than for girls.

Our results underline the importance of providing children with meals nutritionally adequate and suggest that the energy content of children's meals should be increased during particular conditions, like a summer camp, and continuously monitored to assure an adequate energy intake to cover the energy requirements during both school and summer camp days.

\section{Conflict of Interest}

There is no conflict of interest. 